\title{
STATUS OF THE SNS CRYOMODULE TEST*
}

\author{
S. Kim, I. E. Campisi, F. Casagrande, M. Crofford, Y. Kang, Z. Kursun, D. Stout, and A. \\ Vassioutchenko ORNL, Oak Ridge, TN 37831, U.S.A.
}

\begin{abstract}
The cryomodule tests are on going to have better understandings of physics as a whole and eventually to provide safe and reliable operation for neutron production. Some features are revealed to be interesting issues and need more attentions than expected, such as operating condition, collective effects between cavities, HOM coupler issues, end-group stability, cavity-coupler interactions, and vacuum/gas physics, waiting for more investigations. Up to now SNS cryomodules were mainly tested at $2.1 \mathrm{~K} / 4.4 \mathrm{~K}, 10$ pulse per second (pps) and 30 pps/60 pps tests are under progress. This paper presents briefly the experiences and the observations during tests of cryomodules.
\end{abstract}

\section{INTRODUCTION}

Since April 2006 the SNS Superconducting linac (SCL) has been providing a main acceleration for the neutron production at the output energies ranges from about 850 $\mathrm{MeV}$ to $1 \mathrm{GeV}$ [1].

Recently $60-\mathrm{Hz}$ tests with all cavities on in a cryomodule have been done for the first time. As repetition rates go higher, more collective behaviors (affecting neighboring cavities) and thermal effects are observed due to mainly field emissions starting from about $7 \mathrm{MV} / \mathrm{m}$. Achieving the safe limits and understanding of the limiting conditions are the most important factors to provide a reliable operation of cryomodules. Fig. 1 is the statistics of operating and limiting gradients achieved from $60-\mathrm{Hz}$ cryomodule tests.

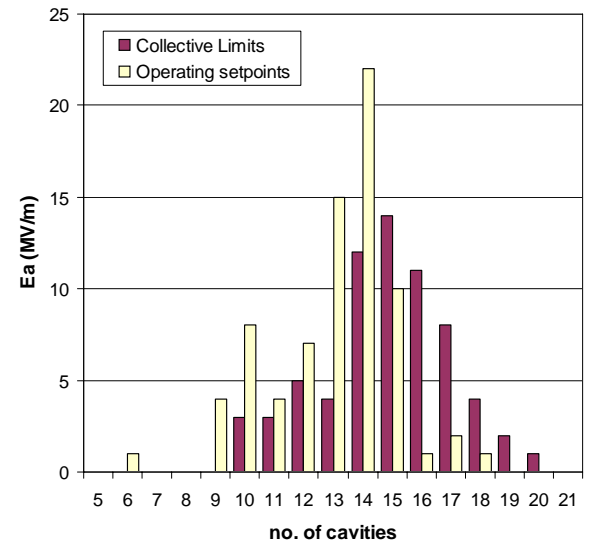

Figure 1. Statistics of limiting gradients and operating gradients from the test results at $60 \mathrm{~Hz}, 1300 \mathrm{us}$ RF pulse.

\section{CRYOMODULE TEST}

\section{Operating Condition}

The transient nature of pulsed operation allows larger

\footnotetext{
* SNS is managed by UT-Battelle, LLC, under contract DE-AC0500OR22725 for the U.S. Department of Energy

"kimsh@ornl.gov
}

variation of temperatures within the niobium surface, so that during the pulse the surface resistance of the material can change substantially. The operating conditions of the SNS SRF cavities were reviewed and cavity parameters and their interplay were reevaluated, giving details on the possible range of operating parameters which can be achieved at SNS in pulsed conditions [2]. Though the nominal operating gradients of the SNS SRF cavities are relatively low, due mainly to the pulsed nature of the SNS operation and the relatively low operating frequency the cavities can still be operated at $4.2 \mathrm{~K}$ up to the critical field. Both calculations and experiments tell us that there's no difference in performances of the SNS SRF cavities as long as the operating temperature is lower than $4.5 \mathrm{~K}$.

\section{Cavity-coupler Interaction}

Cold cathode gauges (CCGs) and electron probes installed to protect the coupler window show strong evidences of not only multipacting but also interactions between cavity field and wave patterns at the power coupler. Good fractions of cavities show double-spike signals from the electron probe as in Fig. 2. This double spike appears at the same time at a certain cavity field or higher. The onset moments of spikes correspond to moments of the full traveling waves at the coupler (Fig. 3) but RF power levels of two spikes at the coupler are different.

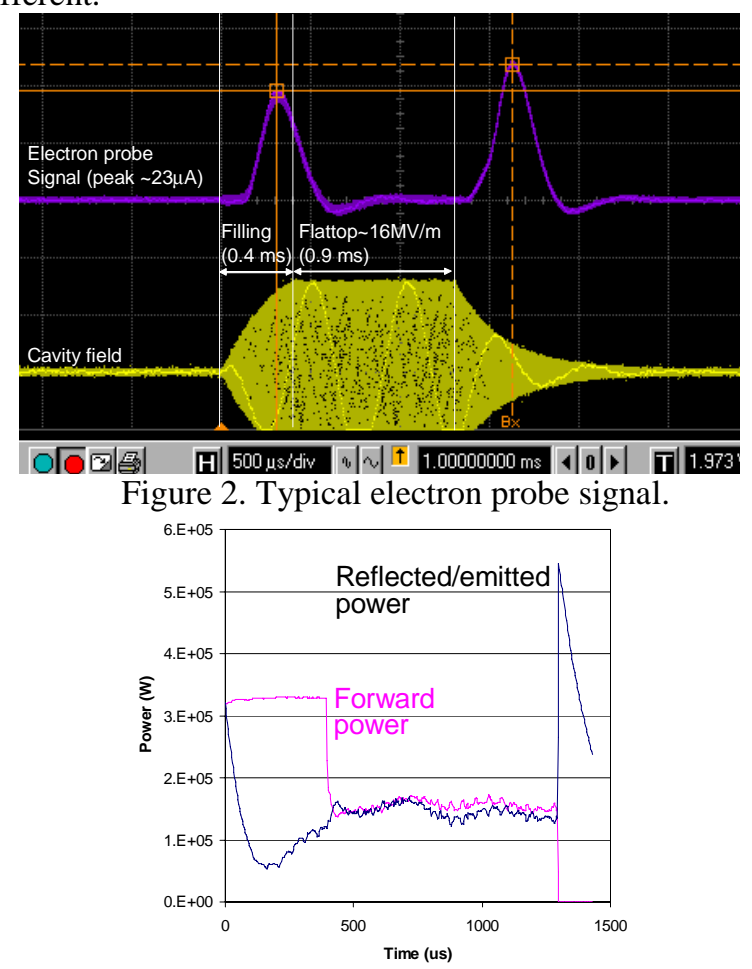

Figure 3. Typical waveforms of forward and reflected powers in the SNS SRF cavities. 


\section{Field Emission and Collective Behaviors}

The measured average gradient of field emission (FE) thresholds is $10 \mathrm{MV} / \mathrm{m} \pm 3 \mathrm{MV} / \mathrm{m}$. The operating gradients are somewhere between the FE threshold and $90 \%$ of the limiting gradients. One interesting observation is that radiation patterns from some cavities do not show cavity field shape (Fig. 4). It has spikes at the end of RF pulses and/or during the cavity filling as shown in Fig. 5. At those moments when radiation waveforms are showing spikes, the power flowing through the fundamental power coupler has full standing wave pattern that is very consistent with the onset moments of electron probe signal (Fig. 3), and at the same moments we observed spikes from signals of HOM couplers in some cavities.

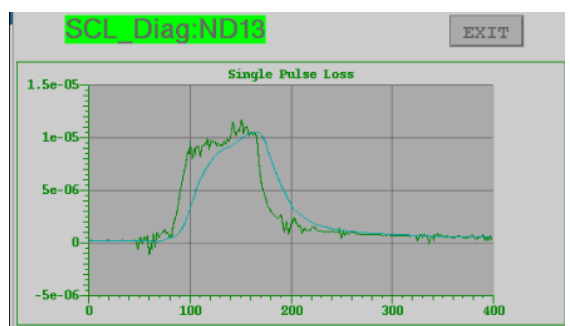

Figure 4. Radiation signal with cavity field shape.
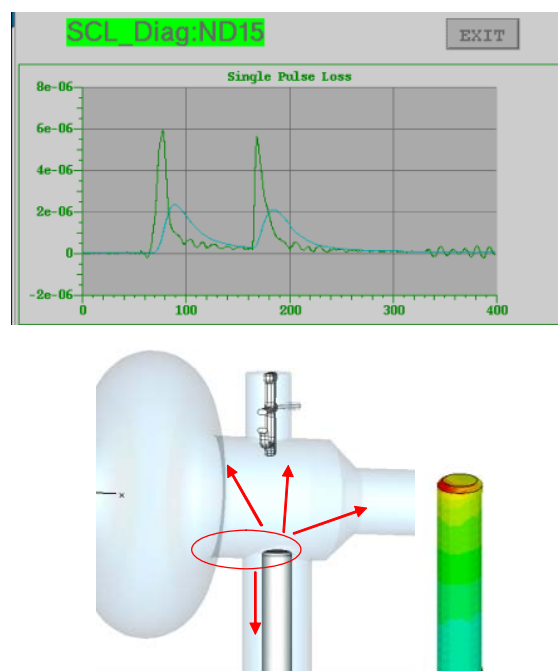

Figure 5. Radiation signal with double spikes shape (top) and schematics of electron activities that could generate both radiations and electron probe signals (Fig. 3) with double spike.

The FE is the major limiting factor especially in high beta cavities, which leads lower operating gradients in most of high beta cavities (average operating gradient $12.5 \mathrm{MV} / \mathrm{m})$ than the design one $(15.8 \mathrm{MV} / \mathrm{m})$. The effects of FE on the achievable gradients and the safe operating gradients are clearer at higher repetition rates including collective behaviors. It is observed in many cryomodules that the landing place of electrons depends on not only the field strength but also relative phases and amplitudes between neighboring cavities as shown in Fig. 6. Also when electrons hit any intermediate temperature region $(<20 \mathrm{~K})$ in the cavity string, big bursts of gas are observed, which makes vacuum trips and redistributions gas and changes in cavity and/or coupler conditions.
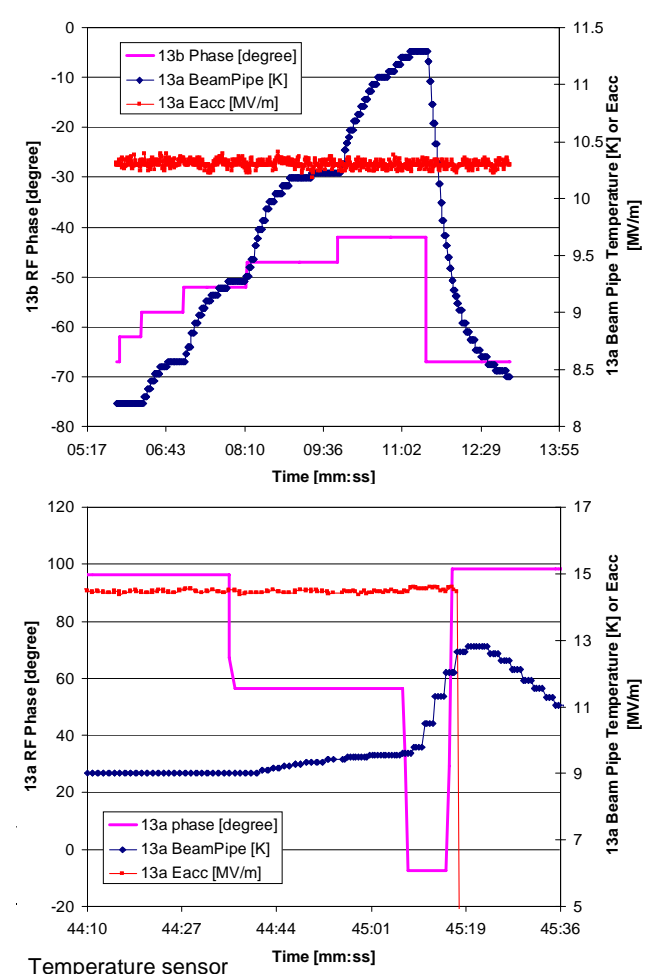

Temperature sensor Time [mm:ss]

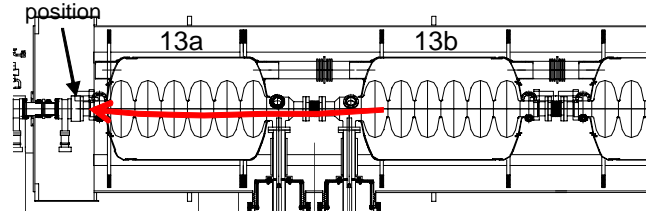

Figure 6. Excursions of the beam pipe temperature of a cavity by changing its own RF phase and the neighboring one's RF phase.

\section{Lorentz Force Detuning}

Observed detuning agrees with expectations in a good fraction of cavities. The detuning coefficients are about $3 \sim 4 \mathrm{~Hz} /(\mathrm{MV} / \mathrm{m})^{2}$ and $1-2 \mathrm{~Hz} /(\mathrm{MV} / \mathrm{m})^{2}$ in medium and high beta cavities respectively. Some cavities show higher detuning coefficients at higher repetition rates as shown in Fig. 7, implying dynamic resonance condition.

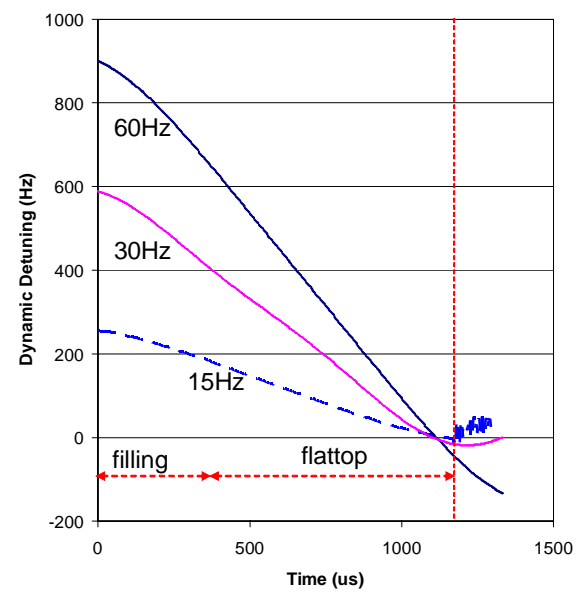

Figure 7. Lorentz force detuning at various repetition rates observed in some of the SNS high beta cavities. In this example the accelerating gradient is $12.7 \mathrm{MV} / \mathrm{m}$.

T07 Superconducting RF 


\section{Cryogenic Load}

Every cavity has a heater to keep the helium pressure constant (ex. 40 mbar at $2 \mathrm{~K}$ operation). The total cryogenic loads from RF operation could be quite accurately extracted from the differences of total heater power compensation.

We tested 3 sets of gradients (Set 1; gradients less than FE threshold, Set 2; gradients established based on individual limits, Set 3; gradients established based on collective limits). The average gradients of Set 3 are slightly less than those of Set 2 by about $0.5 \mathrm{MV} / \mathrm{m}$ and 5 more cavities are incorporated in Set 3, which results in about same output energy. Fig. 9 shows how sensitive the amounts of field emission are depending on gradient sets. The cryogenic loads are quite linear with duty a factor, which implies recovery during the gap between pulses is good enough.

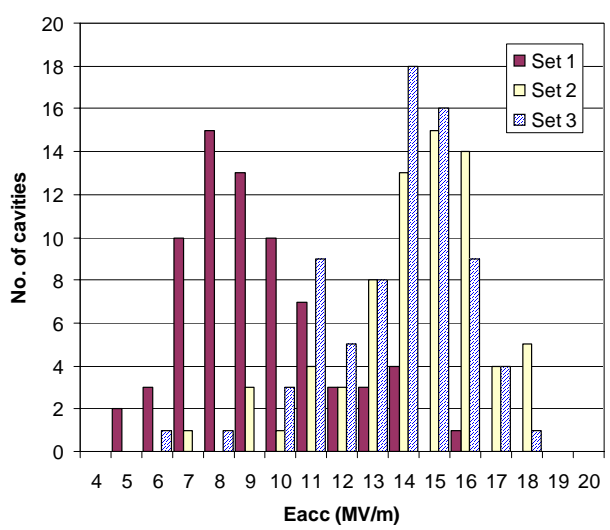

Figure 8. Gradient sets in Fig. 9.

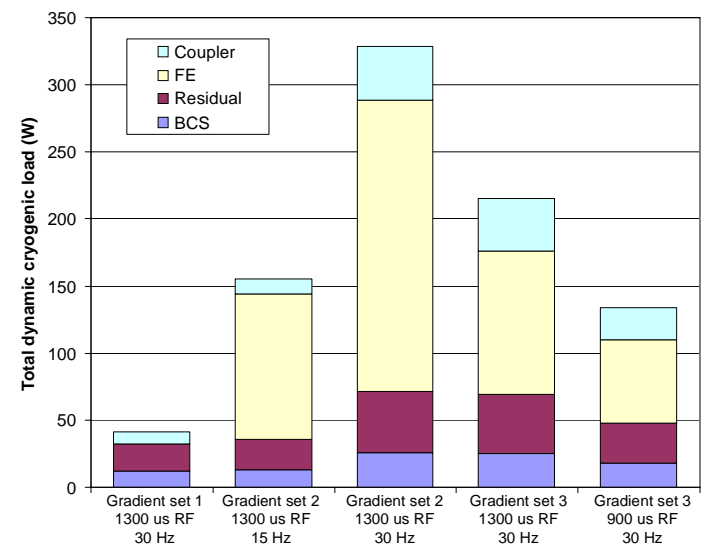

Figure 9. Classification of cryogenic loads from RF.

\section{SUB-COMPONENT CONCERNS}

\section{HOM Couplers}

About $15 \%$ of installed cavities in the SNS SCL are showing abnormal signals through HOM feedthroughs. Observations and physical conditions at around HOM couplers imply that HOM coupler failures and/or degradations seem to be a result of electron activities originated by combinations of multiple causes such as electromagnetic field at the HOM coupler, Multipacting, field emission, and even a gas discharge.

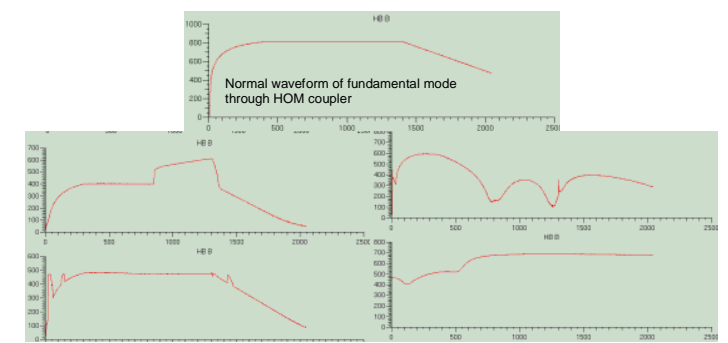

Figure 10. Various waveforms from HOM couplers while running RF only. Top; normal waveform of fundamental mode through HOM coupler ( $y$-axis has logarithmic scale). Left; Multipacting. Right middle; Repetition rate dependent signal. Right bottom; discharge-like signal.

\section{Protection Systems}

CCGs are currently installed to protect the vacuum window of the fundamental power coupler (FPC). Some CCG's have been showing uncertainties of vacuum readings and deterioration, which is possibly caused by damaged electrode for field emission in CCG, which is giving rise to reliability issues as an interlock and is even impeding operations of SRF cavities. Fig. 11 shows the excursions of CCG readings and electron probe signals.

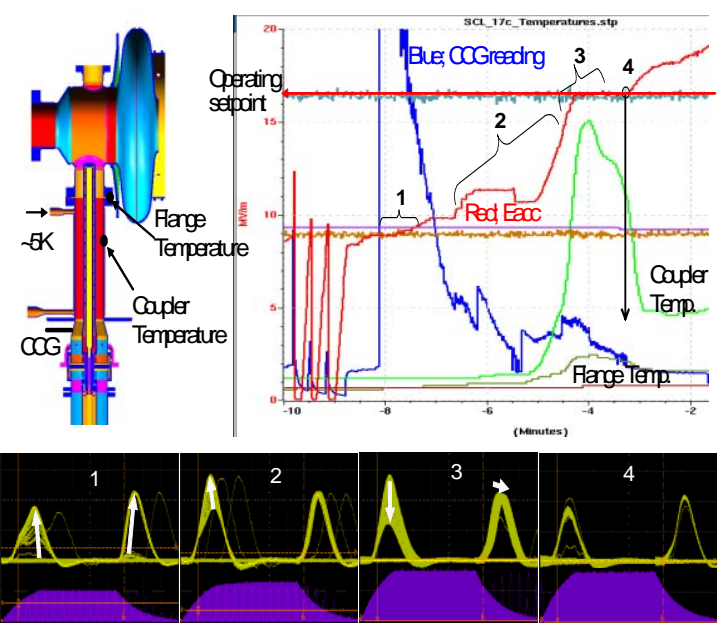

Figure 11. Comparisons of signals between CCG and electron probe while turning on a cavity.

\section{SUMMARY}

From the series of tests and operational experiences more understandings of cavities physics and limiting conditions of the system in pulsed mode are being obtained. We are focusing on reliable, well-balanced and stable operations of cryomodules and supporting systems as a whole. Additional/periodic tests and reevaluations of cryomodules are planned in the tunnel and in the test cave that is planned to be operational end of this year.

\section{REFERENCES}

[1] I.E.Campisi, et al, "Status and performance of the Spallation Neutron Source superconducting linac," PAC’07, Albuquerque, NM, June 2007.

[2] S.-H Kim and I.E.Campsi, "Thermal stability and optimal operating parameters for the Oak Ridge Spallation Neutron Source superconducting linear accelerator,” PRST-AB, 10, 032001 (2007).

T07 Superconducting RF 